\title{
Seizure Detection Using the Phase-Slope Index and Multichannel ECoG
}

\author{
Puneet Rana, \\ Department of Electrical and Computer Engineering, University of Wisconsin-Madison, Madison, \\ WI 53715 USA (rana.puneet@gmail.com).
}

\begin{abstract}
John Lipor,
Department of Electrical and Computer Engineering, King Abdullah University of Science and Technology, Thuwal 23955-6900, Saudi Arabia (john.lipor@gmail.com).
\end{abstract}

\section{Hyong Lee,}

Google Inc., Mountain View, CA 94043 USA (hyongel@gmail.com).

\section{Wim van Drongelen,}

Department of Pediatrics, Department of Neurology, Committee on Computational Neuroscience, and the Computation Institute, University of Chicago, Chicago, IL 60637 USA

(wvandron@peds.bsd.uchicago.edu).

Michael H. Kohrman, and

Department of Pediatrics, Department of Neurology, and the Pediatric Epilepsy Center, University of Chicago, Chicago, IL 60637 USA (mkohrman@peds.bsd.uchicago.edu).

\section{Barry Van Veen ${ }^{*}$ [Fellow, IEEE]}

Department of Electrical and Computer Engineering, University of Wisconsin-Madison, Madison, WI 53715 USA vanveen@engr.wisc.edu

\section{Abstract}

Detection and analysis of epileptic seizures is of clinical and research interest. We propose a novel seizure detection and analysis scheme based on the phase-slope index (PSI) of directed influence applied to multichannel electrocorticogram data. The PSI metric identifies increases in the spatiotemporal interactions between channels that clearly distinguish seizure from interictal activity. We form a global metric of interaction between channels and compare this metric to a threshold to detect the presence of seizures. The threshold is chosen based on a moving average of recent activity to accommodate differences between patients and slow changes within each patient over time. We evaluate detection performance over a challenging population of five patients with different types of epilepsy using a total of 47 seizures in nearly $258 \mathrm{~h}$ of recorded data. Using a common threshold procedure, we show that our approach detects all of the seizures in four of the five patients with a false detection rate less than two per hour. A variation on the global metric is proposed to identify which channels are strong drivers of activity in each patient. These metrics are computationally efficient and suitable for real-time application.

\section{Keywords}

Epilepsy; multichannel electrocorticogram (ECoG); phase-slope index (PSI); seizure detection; seizure evolution

\footnotetext{
(C) 2012 IEEE

*vanveen@engr.wisc.edu.
} 


\section{Introduction}

ONE of the unresolved mysteries in neuroscience is information flow and processing in the immensely complex networks of the brain. Even an obvious disruption of this flow, such as that which occurs in pathology like epilepsy is poorly understood. Although the EEG or electrocorticogram $(\mathrm{ECOG})$ during epileptic seizures seems simple compared to normal interictal activity, the transition into and out of the seizure state is sudden and unexplained. The large variety of epilepsies complicates matters and indicates that multiple mechanisms may underlie epileptogenesis, all culminating in a final common phenomenon-the seizure [1], [2]. Accurate detection of seizures is not only a prerequisite for better understanding seizure generation; a reliable automated seizure detector would also be a boon for clinicians who must find epileptiform activity in long-term recordings. In some cases, localizing the seizures is simple because they occur frequently (several per hour or day), but in other cases, occurrence is rare (one seizure per day, week, or month). Finally, a reliable detection of these events is also valuable for the evaluation of efficacy of therapeutic intervention.

Early methods to detect seizure activity were driven by the practical need to find epileptiform events in clinical recordings. These methods are based on single-channel analysis and detection of specific features in the signal [3], [4]. Variations on these methods are still employed (e.g., Kobayashi et al. [5]). A few decades later, the focus shifted from detection of seizure onset to early detection and even anticipation of seizures, typically using nonlinear dynamical system analysis techniques, for example, the signals are embedded and examined for a change in correlation dimension, entropy, or Lyapunov exponent around seizure onset [6]-[13]. The underlying hypothesis here is that the seizure onset process is deterministic and low dimensional so that it can be predicted. In cases where the onset has a stochastic component, such a prediction would be, of course, impossible [14]. The initial optimism about the prediction power of these techniques was based on the analysis of relatively short epochs of data-several hours at best. Current investigations of long-term data sets ( $24 \mathrm{~h}$ and longer) have shown that anticipation procedures are sensitive to many events besides seizure onset, thereby significantly reducing the specificity of these techniques [15].

Multichannel analysis is an alternative to single-channel examination. Brain activity balances similarity between channels associated with propagation of information and dissimilarity associated with high entropy/information content. To this end, several multichannel methods have been applied to study clinical and experimental seizure activity using a variety of metrics: cross correlation, coherence, Granger causality, transfer functions, and several nonlinear equivalents [16]-[21]. Although these studies have revealed that such metrics may change with seizure state, the search for techniques to follow information processing in the brain is far from completed. Because of the relationships between channels displayed during overt seizure propagation, quantification of channel relations is an ongoing research problem. Often, combined or multiple step approaches are used to assess dependence between areas [22]-[24]. The challenges with multichannel analysis are keeping computational load within reasonable limits and depicting the results in a manner that they can be easily interpreted. The latter is absolutely critical in a clinical setting.

The purpose of this study is to quantify channel interrelationships of brain electrical activity using a novel single-step approach for the detection of spatiotemporal patterns in the multichannel data based on the phase-slope index (PSI) [25]. The PSI computes a directed, pairwise measure of interaction between two channels. We test the hypothesis that the sum of all pairwise PSI values for all channels increases during seizure propagation and show 
that it is a sensitive measure of seizure activity. The net PSI from each channel to all others as a function of time quantifies the flow of information originating in each channel.

A unique strength of this paper is the design of methods and evaluation of performance on long-term recordings from a very challenging population of five pediatric patients with different types of neocortical epilepsy. The five patients selected for this study were already prescreened for epilepsy surgery, and therefore suffered from a combination of frequent and intractable seizures. Abnormal background activity and subclinical events are common in these records and pose a significant challenge for any automated detection scheme. It is difficult for even experienced clinicians to distinguish these events from full clinical seizures without the aid of simultaneous recorded video. Between 41 and $63 \mathrm{~h}$ of data are analyzed for each patient to evaluate seizure detection performance and information flow. A significant feature of our proposed PSI approach is computational efficiency. Our algorithms run faster than real time- $24 \mathrm{~h}$ of data take less than $24 \mathrm{~h}$ to process-on a PC using MATLAB. The information relationships represented by the PSI metrics are also simple to analyze.

Our PSI-based metrics of interaction are introduced in Section II and the data are described in Section III. The results are presented in Section IV and a discussion concludes the paper in Section V.

\section{Methods}

\section{A. Phase-Slope Index}

The PSI was first introduced in [25] where it was also shown to be less sensitive to arbitrary mixtures of independent noise sources than the Granger causality measure [26]. The central idea behind the PSI measure of causal influence is that the cause precedes the effect in time and thus the slope of the phase of the cross spectrum between two signals reflects the direction of influence. Consider signals $z_{i}[n]$ and $z_{j}[n]$. The cross spectrum between them is defined as follows:

$$
S_{i j}(f)=\mathbb{E}\left[Z_{i}(f) Z_{j}^{*}(f)\right]
$$

and the complex coherence is

$$
C_{i j}(f)=\frac{S_{i j}(f)}{\sqrt{S_{i i}(f) S_{j j}(f)}} .
$$

The unnormalized PSI metric is defined using complex coherence as follows:

$$
\tilde{\Psi}_{i j}=\operatorname{Imag}\left(\sum_{f \in F} C_{i j}^{*}(f) C_{i j}(f+\delta f)\right)
$$

where $F$ is the frequency band of interest and $\delta f$ is the frequency resolution. It is straightforward to show that $\tilde{\Psi}_{i j}$ measures a weighted sum of the slopes of the phase between $z_{i}[n]$ and $z_{j}[n]$ over the band $F[25]$. This measure is normalized by its standard deviation to obtain a metric $\Psi_{i j}$ that can be used to determine whether causal influence from $Z_{i}[n]$ to $Z_{j}[n]$ is significant: 


$$
\Psi_{i j}=\frac{\tilde{\Psi}_{i j}}{\operatorname{std}\left(\tilde{\Psi}_{i j}\right)} .
$$

Nolte et al. [25] suggest that absolute values of $\Psi_{i j}$ greater than 2 should be considered significant. We compute $\Psi_{i j}$ using the MATLAB software available in [27].

\section{B. Metrics of Connectivity}

Our hypothesis is that the level of causal interactions in the brain are much higher during a seizure than an interictal time segment. This hypothesis is consistent with the wide spreading of highly correlated activity that characterizes seizures. We use the normalized PSI (4) to construct a metric of global interaction between measured channels over a given time segment, as described in the following, and then use this metric to automatically identify the onset of seizure activity.

Let $x_{j}[n]$ and $x_{j}[n]$ denote the measured signal in channels $i$ and $j$, respectively, where $i, j \in$ $\{1,2, \ldots, M\}$. Define $z_{i}^{k}[n]$ as the $k$ th segment or interval of $x_{i}[n]$ of length $N$ samples

$$
z_{i}^{k}[n]=\left\{\begin{array}{ll}
x_{i}[n], & \text { if }(k-1) N<n<k N \\
0, & \text { otherwise }
\end{array} .\right.
$$

Note that this definition assumes that successive segments do not overlap. It is straightforward, but more complex notationally, to construct $z_{i}^{k}[n]$ from overlapping sections of $x_{j}[n]$. Now let $\Psi_{i j}^{k}$ be the PSI (4) computed using $z_{i}^{k}[n]$ and $z_{j}^{k}[n]$. Then, the net outward PSI from channel $i$ during the $k$ th time segment is given as follows:

$$
\Lambda_{i}^{k}=\sum_{\substack{j=1 \\ j \neq i}}^{M} \Psi_{i j}^{k} 1_{\left\{\Psi_{i j}^{k} \geq 2\right\}}
$$

where

$$
1_{\left\{\Psi_{i j}^{k} \geq 2\right\}}= \begin{cases}1, & \text { if } \Psi_{i j}^{k} \geq 2 \\ 0, & \text { otherwise }\end{cases}
$$

is used to only include significant PSI values in the net causal influence of a channel $i$ during a particular segment $k$. We measure the global level of interaction in the $k$ th segment of data as follows:

$$
\Lambda^{k}=\sum_{\substack{i, j=1 \\ i \neq j}}^{M} \Psi_{i j}^{k} 1_{\left\{\Psi_{i j}^{k} \geq 2\right\}} .
$$

In other words, we form $\Lambda^{k}$ as the sum of significant PSI values over all pairwise interactions.

We compare the detection performance of the PSI-based $\Lambda^{k}$ to that of a simple moving average of the global power. Define the global power in the kth segment of data as follows: 


$$
P^{k}=\sum_{i=1}^{M} \sum_{n=(k-1) N+1}^{k N}\left(z_{i}^{k}[n]\right)^{2}
$$

Power metrics have been used for seizure detection, e.g., [28].

\section{Detection of Seizures}

We declare the presence of a seizure in segment $k$ if $\Lambda^{k}$ exceeds a threshold $\Gamma^{k}$. Here we choose $\Gamma^{k}$ as a moving average of $\Lambda^{k}$ plus a certain number of standard deviations, i.e.,

$$
\Gamma^{k}=\bar{\Lambda}^{k}+c * \sigma_{\Lambda^{k}}
$$

where the moving mean $\Lambda^{-k}$ and the standard deviation $\sigma_{\Lambda} k$ are

$$
\begin{gathered}
\bar{\Lambda}^{k}=\frac{1}{L} \sum_{i=k-L}^{k-1} \Lambda^{i} \\
\sigma_{\Lambda^{k}}=\left(\frac{1}{L-1} \sum_{i=k-L}^{k-1}\left(\Lambda^{i}-\bar{\Lambda}^{k}\right)^{2}\right)^{\frac{1}{2}} .
\end{gathered}
$$

The threshold is determined using the $L$ most recent values of $\Lambda^{k}$ to identify significant short-term changes in global interactivity as seizures. This adjusts for longer term evolution in baseline global interactivity associated with changes in state and accounts for differences between patients. Metrics $\Lambda^{k}$ from segments that exceed $\Gamma^{k}$ are not used to calculate future thresholds; therefore, the threshold is not biased upward by the presence of seizure activity. Also, consecutive segments for which $\Lambda^{k}$ exceeds $\Gamma^{k}$ are counted as a single detection.

Similarly, we define the threshold $\Theta^{k}$ for global power based seizure detection as follows:

$$
\Theta^{k}=\bar{P}^{k}+d * \sigma_{p k}
$$

where the moving mean $\bar{P}_{P}^{k}$ and standard deviation $\sigma_{P}$ are computed using the $L$ most recent values of $P^{k}$ analogously to (10) and (11).

We expect a time delay between seizure initiation and PSI or power-based detection for three reasons: 1) Detection cannot occur until the end of the $N$-sample segment used to compute $\Lambda^{k}$ or $P^{k}$. (2) Use of nonoverlapping segments quantizes possible detection times to multiples of $N$. 3) The clinically identified seizure onset is determined using a noncausal analysis of the record and generally there is a time delay to subsequent development of detectable increases in global power or interactivity. Hence, we declare a seizure to be correctly detected if $\Lambda^{k}$ or $P^{k}$ exceeds the threshold within 2 min after the identified initiation time. All other instances of $\Lambda^{k}$ or $P^{k}$ exceeding the threshold are false positives.

We compute the seizure detection percentage based on the ratio of correctly detected seizures to the number of actual seizures. The number of false detections per hour is also computed as the ratio of false positives to the total number hours in the data. 


\section{Seizure Evolution Analysis}

The nature of causal influence during seizures is assessed for each patient by averaging the net PSI from each channel to all other channels over all seizures. Let $S$ denote the set of clinically identified seizure initiation time indices and define

$$
\tilde{\Lambda}_{i}^{l}=\frac{1}{|S|} \sum_{k \in S} \sum_{\substack{j=1 \\ j \neq i}}^{M} \Psi_{i j}^{k+1} 1_{\left\{\Psi_{i j}^{k+1} \geq 2\right\}} .
$$

This metric represents the net causal influence of the $i$ th channel as a function of time $I$ relative to seizure initiation averaged over all seizures. Hence, this metric identifies the common spatiotemporal PSI features across seizures.

\section{Data}

\section{A. Description}

All data were acquired from epilepsy surgery candidates of Comer Children's Hospital at the University of Chicago. Patient selection was based on the presence of multiple seizures as well as the availability, quality, and duration of recordings; no other screens, except for an age criterion excluding patients younger than three years old, were applied. In the patient group, only two patients (patients 1 and 2) are typical mesial temporal lobe cases, the other three show more complex pathology. The clinical epileptologist of the team (M. H. Kohrman) determined seizure onset times and leading channels using visual analysis of the $\mathrm{EEG} / \mathrm{ECoG}$ and the audiovisual recording. The ECoG recorded for each patient was reviewed and obvious equipment-related artifacts, such as the start and stop of recording or data from loose electrodes, were excluded; otherwise, the ECoG records were analyzed in their entirety. A summary of the five selected patients are given in Table I.

\section{B. Acquisition and Preprocessing}

The EEG and ECoG time series were recorded with a bandwidth of $0.5-100 \mathrm{~Hz}$, and digitized at 400 samples/s with a 12-bit A/D converter using a BMSI 6000 unit (Cardinal Health, Dublin, $\mathrm{OH})$. All channels used a common scalp reference $(\mathrm{CPz})$. As a part of the preoperative evaluation, the activity of 128 channels was recorded as a combination of intracranial (Radionics Medical Products Inc., Burlington, MA) and scalp electrodes for each patient. Intracranial electrodes were placed on the cortical surface in locations dependent on expected seizure onset location. Data from the scalp EEG electrodes were not used in this study. For patients 4 and 5, the set of intracranial electrodes used was changed during the data collection interval for clinical considerations.

The time series data sampled at $400 \mathrm{~Hz}$ was passed through a high-pass second-order Butterworth filter with cutoff frequency of $1 \mathrm{~Hz}$ in order to discard LF noise, and then lowpass filtered to $50 \mathrm{~Hz}$ prior to downsampling the data to a $100 \mathrm{~Hz}$ sampling rate. Downsampling was performed to limit the memory and computational costs for this very large dataset.

\section{Results}

For the examples and the results in this paper, we have considered $1-11 \mathrm{~Hz}$ as the frequency band of interest $F$ in (3) because the vast majority of seizure power lies below $11 \mathrm{~Hz}$. The frequency resolution $\delta f$ in (3) is chosen as $0.25 \mathrm{~Hz}$. 


\section{A. Effect of Window Size on PSI Consistency}

We first used the simulated scenario depicted in Fig. 1 to identify appropriate segment lengths $N$ in (5) based on the sensitivity of the PSI (4) to a 1-s temporal shift of the input signals. We evaluated a case where the PSI should be large — between $z_{1}[n]$ and $z_{2}[n]$ — and another case where the PSI should be zero-between $z_{1}[n]$ and $z_{3}[n]$. Note that the IIR allpass filter introduces a non-linear phase shift, that is, non-constant group delay, between $z_{1}[n]$ and $z_{2}[n]$. Five hundred independent simulations were conducted to evaluate the average PSI, the average change in PSI due to a 1-s shift in the signals, and the standard deviation of the PSI change for segment lengths $N$ corresponding to 10, 20, 30, 45, and $60 \mathrm{~s}$. Table II lists these outcomes. The 10-s segment results in the smallest PSI value for $z_{1}[n]$ and $z_{2}[n]$, a mean change of half the average PSI, and the standard deviation of the change that exceeds the mean of the change. The average PSI increases as the segment length increases consistently with the true underlying scenario, and the mean and standard deviation of the change stabilize as the segment length increases. Similar behavior is observed for the case where there is no underlying relationship between the signals. In the subsequent data analysis, we employed 20- and 60-s segment lengths.

\section{B. Seizure Connectivity Measured by PSI}

Fig. 2 graphically depicts significant PSI values $\Psi_{i j}$ with $i, j=1,2, \ldots, 88$ defined in (4) for 60 -s segments of interictal and ictal data from patient 2 . The figure shows a dramatic increase in causal influence between channels during the seizure. This characteristic is representative of all patients and seizures in this study and motivates the global PSI metric $\Lambda^{k}(7)$ as an indicator of seizure activity. Fig. 3 depicts $\Lambda^{k}$ and the threshold $\Gamma^{k}(9)$ for 1600 min of recording (about $26.7 \mathrm{~h}$ ) from patient 1 using 20-s segments. Seizure events show a dramatic increase in $\Lambda^{k}$. The threshold shown is computed using $c=8$ in (9) and $L=90$ segments (30 $\mathrm{min}$ ). This threshold results in one falsely detected seizure in the record, as shown in the figure.

\section{Seizure Detection Performance}

We evaluate the performance of our PSI-based automated seizure detection approach on the five patients using seizure detection percentage and false detections per hour. These parameters were computed for a range of threshold values for each patient by varying the constant $c$ in (9) between 0 and 25. The two graphs shown in Fig. 4 illustrate the seizure detection percentage and the false detections per hour for patients 2 and 5 . The general trend of these graphs was the same for all patients: at low thresholds, all the seizures are detected but at the cost of a greater number of false detections. The fall in false detections as $c$ increases is steeper than that of the seizure detection percentage. This is further highlighted in Table III where the performance parameters are listed for the maximum $c$ where $100 \%$ of the seizures were detected. The numbers for both 20 - and 60-s segment lengths are displayed using 30 min of past PSI values to calculate the threshold, i.e., using $L=90$ segments and $L$ $=30$ segments, respectively, in (9). The seizures from patient 1 have the greatest contrast with interictal activity since all of them are detected using a threshold based on 17 and 21 standard deviations for 20- and 60-s segments, respectively. At these thresholds, there are no false detections. The seizures of patient 3 have the least contrast. The maximum thresholds for detection of all of patient 3's seizures are based on 2.7 and 0.8 standard deviations for 20- and 60-s segments, respectively. The rate of false detection is still quite high at these thresholds, exceeding 7 per hour. With 20-s segments, all seizures but one of patient 3's are detected using a threshold based on 4.8 standard deviations, and the corresponding rates of false detection are less than 1 per hour, excluding the rate for patient 4 , which is at 1.9 per hour. Other than patient 1 , fairly large thresholds - greater than 10 standard deviations-are required to achieve zero false detections. 
We investigated the nature of false detections by asking a board-certified neurologist (M. H. Kohrman) to examine examples of the EEG of false detections in two patients, 2 and 3 . The neurologist reviewed 13 EEG segments of $1 \mathrm{~min}$ before and after the false detection, as well as the 1 min segment that was erroneously flagged as a seizure. The neurologist concluded that the false detection segments fell into one of four categories: 1) Subclinical events; 2) periods of constant background activity that indicated abnormal EEG but not a subclinical event; 3) equipment artifact; and 4) no abnormal activity. Categories 1 and 2 dominated false detections. Fig. 5 provides examples of cases 1 and 3. As epilepsy surgery candidates, the subjects suffered from a combination of frequent and intractable seizures. Abnormal background and subclinical events are common in the record, and present a challenge for any automated system; indeed, without the help of video recorded simultaneously with the EEG, it is often difficult for even experienced clinicians to distinguish between these events and full clinical seizures.

Table IV depicts detection performance using 20-s segments for both PSI and power methods. The largest common threshold that resulted in the detection of all seizures for four out of five patients was chosen. This resulted in $c=4.8$ for the PSI method and $d=2.1$ for the power method. The detection performance is comparable: the PSI method only missed 1 of 15 seizures in patient 3 , while the power method missed 1 of 15 in patient 5 . However, the PSI method yields dramatically better false detection performance-between 0.15 and 1.9 per hour-compared to 10 to 19 per hour for the power method. We note that false detection rates decrease for 60 -s segments, ranging from 0.13 to 0.43 for the PSI method and 1.4 to 3.7 for the power method. However, this reduction is associated with detecting fewer true seizures in one of the patients and increased detection latency.

The average latency of detected seizures using 20-s segments and a threshold based on 4.8 standard deviations for the PSI method and 2.1 standard deviations for the power method is shown for each patient in Fig. 6. The error bars in the graph denote one standard deviation. We define the delay as the time difference between end of the segment in which the seizure is first detected and the seizure onset identified by the clinician. For example, a delay of $25 \mathrm{~s}$ implies that the seizure was detected using the data from 5 to $25 \mathrm{~s}$ after the identified start of the seizure. Note that seizures could start at any time within a segment-we did not synchronize the segment locations to the seizure onset times. Furthermore, the onset times identified by the epileptologist are determined in a noncausal manner by going backward in time from well-developed seizure activity to find the very first indications of a seizure. Hence, the latencies in Fig. 6 represent the delay in seizure detection that would result in a real-time clinical application. Except for patient 3 and the power method of patient 4, seizures are generally detected with delay on the order of the segment length. The standard deviation of the power method latencies is considerably larger than those of the PSI method.

\section{Seizure Evolution Analysis}

The average spatiotemporal evolution of all seizures for each patient evaluated according to (13) is shown in Fig. 7 as the average outward PSI flow from each channel as a function of time. The values of $l$ in (13) correspond to the segments from $1 \mathrm{~min}$ prior to $3 \mathrm{~min}$ after the seizure initiation. For patients 4 and 5, the electrodes were changed during the recording interval. We only display the spatiotemporal evolution for patient and electrode configurations in which at least five seizures are available. The dashed horizontal lines correspond to the clinically identified leading channels. In general, there is correspondence between the leading channels and the channels with large postseizure PSI. Table V compares the leading channels to the five channels with maximum PSI summed over time, again for patient and electrode configurations in which at least five seizures are available. The channels with largest PSI include or are located near the leading channels in many cases. 


\section{DISCUSSION}

The PSI is sensitive to interactions involving simple time delays or nonconstant group delay in a portion of the frequency band being analyzed, as demonstrated by the results in Fig. 1 and Table II. The minimum usable data segment length for PSI computation is governed in part by the amount of data required to reliably estimate the cross spectrum. We employed simulated signals to evaluate PSI consistency for a $1-11 \mathrm{~Hz}$ analysis bandwidth and $100 \mathrm{~Hz}$ sampling rate with respect to 1-s shifts in the signals. Consistency with respect to time shifts of the signals is a relevant metric since in this application the seizure onset will naturally vary with respect to the segment location. Other tests for consistency may give slightly different conclusions, and such simulations should only be considered as guides, given the impossibility of simulating realistic scenarios. The level of consistency decreased fairly rapidly with less than 20-s segment lengths and became very stable as the segment length approached $60 \mathrm{~s}$. Consequently, we evaluated detection performance for both 20- and 60-s segment lengths.

The global PSI defined in (7) is sensitive to the widespread increased interaction between channels that occurs during seizure activity. Consequently, it is a suitable metric for detecting seizures. There are multiple parameters that can be adjusted in computing global PSI: the segment length as already discussed, the threshold for which the pairwise PSI is significant, and the method for choosing a global PSI threshold for seizure detection. We adopted the pairwise PSI significance threshold recommended in [25] without further experimentation and have proposed a patient-dependent moving average strategy for choosing the global PSI seizure detection threshold. This strategy is motivated by the observation that the global PSI during interictal conditions varies in mean value and variability across patients and over time. For example, the mean global PSI was observed to be significantly larger for patient 3 than 1, consistent with the frequent nature of subclinical events in this patient. Using a specified number of standard deviations above the mean as a threshold — with both computed using recent data—is one way to standardize the threshold across patients and over time. This strategy is also relatively insensitive to the PSI significance threshold because seizures are identified based on the contrast between interictal and seizure global connectivity. Modest changes in the threshold do not have a significant impact on the contrast.

The PSI method with a common threshold of 4.8 standard deviations and a 20-s segment length gave good performance across our subject population, detecting all but one seizure. Note that the false detection rate decreases very rapidly until about five standard deviations (see Fig. 4) and then tends to flatten out; the number of standard deviations required to obtain zero false detections is quite large (see Table III). Correct detection performance begins to degrade beyond five standard deviations (see Fig. 4, Table III), which suggests that the threshold should be chosen between four and five standard deviations for best overall performance. We also informally explored different data lengths for computing the moving average of the mean and standard deviation, and choose to report results for $30 \mathrm{~min}$ because it gave the best overall results of the variations considered. This time period is long enough to smooth out short-term variations but short enough to respond to longer term changes in brain state, such as sleep versus wake.

Our performance evaluation involved ECoG data from five pediatric patients with different types of epilepsy recorded over time intervals ranging from 41 to $63 \mathrm{~h}$ and containing between 5 and 15 seizures each (see Table I). With the exception of patient 3 , use of 4.8 standard deviations as global PSI threshold resulted in detection of $100 \%$ of the seizures with 20-s segments (see Tables III and IV). The rate of false detections per hour using a common threshold ranges from 0.15 to 1.9 . Note that a false detection rate of 1.9 
corresponds to around two falsely detected seizures every hour on average. In contrast, while global power is able to achieve equivalent detection performance, the rates of false detection are more than a factor of 10 worse, ranging from 10 to 19.

The latency at which seizures are detected is comparable between PSI and power methods (see Fig. 6). The average latency is less than $30 \mathrm{~s}$ when using 20-s segments for the PSI approach, except for patient 3 which is less than $50 \mathrm{~s}$. The power approach latencies are less than $40 \mathrm{~s}$. These results, and the lack of synchronization between segment locations and seizure times, strongly suggest that the global PSI increases significantly very soon after the clinically determined seizure onset. The latency of seizure detection is generally significantly longer with patient 3 , which may also be related to the increased difficulty of seizure detection for patient 3 .

The absolute detection latencies are biased toward positive values due to the causal constraints of real-time detection. First, the epileptologist-marked onset times are identified in a noncausal manner and thus reflect early indications of seizure activity, prior to full seizure development. Second, our reference time is the end of the segment in which the seizure is detected. The latencies reported in Fig. 6 could be reduced by using overlapping analysis segments in (5) as this would effectively synchronize seizure onset times with segment location.

Evaluation of PSI-based seizure detection performance is straightforward compared to the evaluation of spatiotemporal seizure evolution because the clinical information on seizure evolution is limited to the identification of leading channels. Leading channels are associated with seizure initiation while the PSI metric represents net activity over a segment of time once the seizure begins; therefore, differences between the clinical- and PSI-based analysis are expected. However, the five channels with largest PSI outflow included at least one of the leading channels for three out of the five patients (see Table V). Channels neighboring the leading channels also had large PSI outflow in many cases.

The average spatiotemporal evolution depicted in Fig. 7 does not have a clinical baseline for comparison. However, the information contained in this display is informative and potentially useful for understanding seizure dynamics. We observe that significant PSI outflow is limited to a small subset of channels in patients 1 and 2 . The leading channels show peak PSI outflow over a minute after seizure onset, while other channels have large PSI values closer to seizure onset. In contrast, the leading channels for patient 5 show peak PSI outflow in the 20-40 s postseizure interval. The challenge of seizure detection for patient 3 is evident in the relatively diffuse, low level of PSI outflow across the majority of channels. The portion of the evolution within 2 min of seizure onset for patient 4 also consists of very low level PSI outflow, suggesting that there is no consistent outflow pattern across the five seizures analyzed. These differences in evolution may be due to the different types of epilepsy in the patient population. Note that the widespread interaction between channels suggested by Fig. 2 is less apparent in Fig. 7 due to averaging over seizures. Also, the color scale used here diminishes the appearance of weaker PSI values.

The spatiotemporal evolution also complements the detection latency analysis of Fig. 6. In Fig. 7, the segment start times are synchronized to seizure onset. Patients 1, 2, and 5 have well-defined PSI outflow but show very little significant activity in the first $20 \mathrm{~s}$ following the seizure onset. The most significant PSI outflow begins $20 \mathrm{~s}$ or more after seizure onset, even though the seizures are detected with a mean latency of less than $30 \mathrm{~s}$ in the absence of synchrony between segment origins and seizure onset. This observation is likely a consequence of the development of seizure activity after clinically defined onset. 
The PSI is a pairwise metric of directional interactions, and thus is subject to ambiguity between direct and indirect influence. For example, if the PSI from channel A to B and channel $\mathrm{A}$ to $\mathrm{C}$ are both significant, it is not possible to determine whether the influence of channel $\mathrm{A}$ on $\mathrm{C}$ is direct or indirectly mediated through channel $\mathrm{B}$. This ambiguity is a problem for reconstructing network topologies, but is not significant in the application proposed here. The net PSI outflow from a channel represents the total impact of that channel on all other channels, both direct and indirect, and is a sensible measure of net influence.

The PSI-based approach is nonparametric and has several advantages over parametric approaches such as multivariable autoregressive (MVAR) models, which are used to compute Granger causality, directed transfer functions, and partially directed coherence. While the MVAR approach provides much more information about interaction between channels than the bivariate PSI approach, the computational cost and model complexity limit its potential for real-time seizure detection. The number of parameters in an MVAR model is on the order of $L^{2} p$, where $L$ is the number of channels and $p$ is the memory. In the patients considered here, there are approximately $L=100$ channels; therefore, the number of parameters is extremely large even for modest values of $p$. Reliable estimation of MVAR models with such large numbers of parameters requires significant lengths of stationary data and much greater computational load than the PSI approach. Each MVAR model requires estimation and inversion of an $L p$-by- $L p$ covariance matrix.

Computation of the cross spectra required by the PSI approach typically involves averaging the products of discrete Fourier transforms of windowed versions of the signals. The discrete Fourier transforms are efficiently computed using a fast Fourier transform algorithm. Hence, our PSI approach is amenable to real-time computation. Although the datasets were postprocessed in this paper, the results were obtained in faster than real time on a standard desktop PC using MATLAB without any consideration to real-time programming constructs.

The performance of any seizure detector depends, of course, on the data set used for evaluation. As in most studies, we evaluated performance in typical mesial temporal lobe cases (patients 1 and 2, Table I). In these cases, performance was close to perfect (see Tables IV and V) and the channels showing propagation correspond well with the leading channels identified by the epileptologist [see Table V, Fig. 7(a) and (b)]. In addition, we considered more challenging cases (patients 3-5 in Table I) that are typical for pediatric-aged patients. As expected, the performance decreased but remained very good: in two of the three cases (patients 4 and 5), all seizures were detected, while the false detection rate was under two per hour; in the worst case (patient 3), all but one seizure could still be detected at reasonable false detection rate (see Table IV). The spatial relationship between propagating channels and leads identified by the epileptologist was also less strict in the latter three cases [see Table V, Fig. 7(c)-(f)]. In conclusion, the PSI approach proposed herein is effective, computationally efficient, and easy to interpret, and thus shows significant promise for seizure detection and analysis.

\section{Acknowledgments}

This work was supported in part by the National Institutes of Health under award R21EB009749 and the Dr. Ralph and Marian Falk Medical Research Trust. 


\section{References}

[1]. Bancaud J, Henriksen O, Rubio-Donnadieu F, Seino M, Dreifuss FE, Penry JK. Proposal for revised clinical and electroencephalographic classification of epileptic seizures. Epilepsia. 1981; vol. 22(no. 4):489-501.

[2]. Blume WT, Lders HO, Mizrahi E, Tassinari C, VanEmdeBoas W, Engel J. Glossary of descriptive terminology for ictal semiology: Report of the ILAE task force on classification and terminology. Epilepsia. 2001; vol. 42(no. 9):1212-1218. [PubMed: 11580774]

[3]. Gotman J, Gloor P. Automatic recognition and quantification of interictal epileptic activity in human scalp EEG. Electroencephalogr. Clin. Neurophysiol. 1976; vol. 41(no. 5):513-529. [PubMed: 61855]

[4]. Gotman J. Automatic recognition of epileptic seizures in the EEG. Electroencephalogr. Clin. Neurophysiol. 1982; vol. 54(no. 5):530-540. [PubMed: 6181976]

[5]. Kobayashi K, Agari T, Oka M, Yoshinaga H, Date I, Ohtsuka Y, Gotman J. Detection of seizureassociated high-frequency oscillations above 500 Hz. Episepsy Res. Feb.2010 vol. 88(no. 2-3): 139-144.

[6]. Lehnertz K, Elger C. Neuronal complexity loss in temporal lobe epilepsy: Effects of carbamazepine on the dynamics of the epileptogenic focus. Electroencephalogr. Clin. Neurophysiol. Sept.1997 vol. 103(no. 3):376-380. [PubMed: 9305285]

[7]. Martinerie J, Adam C, Le Van Quyen M, Baulac M, Clemenceau S, Renault B, Varela F. Epileptic seizures can be anticipated by non-linear analysis. Nature Med. Oct.1998 vol. 4(no. 10):11731176. [PubMed: 9771751]

[8]. Iasemidis LD, Sackellares J. Chris, Zaveri HP, Williams WJ. Phase space topography and the Lyapunov exponent of electrocorticograms in partial seizures. Brain Topography. 1990; vol. 2:187-201. [PubMed: 2116818]

[9]. Litt B, Esteller R, Echauz J, D’Alessandro M, Shor R, Henry T, Pennell P, Epstein C, Bakay R, Dichter M, Vachtsevanos G. Epileptic seizures may begin hours in advance of clinical onset: A report of five patients. Neuron. Apr.2001 vol. 30(no. 1):51-64. [PubMed: 11343644]

[10]. Iasemiidis, LD.; Sackellares, JC.; Savit, RS. Quantification of hidden time dependencies in the EEG within the framework of nonlinear dynamics. In: Jansen, B.; Brand, M., editors. Nonlinear Dynamical Analysis of the EEG. World Scientific; Singapore: 1993. p. 30-47.

[11]. Le van Quyen M, Adam C, Baulac M, Martinerie J, Varela F. Nonlinear interdependencies of EEG signals in human intracranially recorded temporal lobe seizures. Brain Res. May; 1998 vol. 792(no. 1):24-40. [PubMed: 9593809]

[12]. Lehnertz K, Andrzejak R, Arnhold J, Kreuz T, Mormann F, Rieke C, Widman G, Elger C. Nonlinear EEG analysis in epilepsy: Its possible use for interictal focus localization, seizure anticipation, and prevention. J. Clin. Neurophysiol. May; 2001 vol. 18(no. 3):209-222. [PubMed: 11528294]

[13]. van Drongelen W, Nayak S, Frim D, Kohrman M, Towle V, Lee H, McGee A, Chico M, Hecox K. Seizure anticipation in pediatric epilepsy: Use of Kolmogorov entropy. Pediatr. Neurol. Sep. 2003 vol. 29(no. 3):207-213. [PubMed: 14629902]

[14]. da Silva, F. Lopes; Blanes, W.; Kalitzin, S.; Parra, J.; Suffczynski, P.; Velis, D. Dynamical diseases of brain systems: Different routes to epileptic seizures. IEEE Trans. Biomed. Eng. May; 2003 vol. 50(no. 5):540-548.

[15]. Mormann F, Kreuz T, Rieke C, Andrzejak R, Kraskov A, David P, Elger C, Lehnertz K. On the predictability of epileptic seizures. Clin. Neurophysiol. Mar.2005 vol. 116(no. 3):569-587. [PubMed: 15721071]

[16]. Steriade M, Amzica F. Dynamic coupling among neocortical neurons during evoked and spontaneous spike-wave seizure activity. J. Neurophysiol. Nov.1994 vol. 72(no. 5):2051-2069. [PubMed: 7884444]

[17]. Towle V, Carder R, Khorasani L, Lindberg D. Electrocorticographic coherence patterns. J. Clin. Neurophysiol. Nov.1999 vol. 16(no. 6):528-547. [PubMed: 10600021] 
[18]. Sitnikova E, Dikanev T, Smirnov D, Bezruchko B, Van Luijtelaar G. Granger causality: Corticothalamic interdependencies during absence seizures in WAG/Rij rats. J. Neurosci. Methods. May; 2008 vol. 170(no. 2):245-254. [PubMed: 18313761]

[19]. Wilke C, van Drongelen W, Kohrman M, He B. Neocortical seizure foci localization by means of a directed transfer function method. Epilepsia. Apr.2010 vol. 51(no. 4):564-572. [PubMed: 19817817]

[20]. Cadotte AJ, DeMarse TB, Mareci TH, Parekh MB, Talathi SS, Hwang D-U, Ditto WL, Ding M, Carney PR. Granger causality relationships between local field potentials in an animal model of temporal lobe epilepsy. J. Neurosci. Methods. May; 2010 vol. 189(no. 1):121-129. [PubMed: 20304005]

[21]. Barbero A, Franz M, van Drongelen W, Dorronsoro J, Scholkopf B, Grosse-Wentrup M. Implicit wiener series analysis of epileptic seizure recordings. Proc. IEEE Annu. Int. Conf. Eng. Med. Biol. Soc. Sep.2009:5304-5307.

[22]. Gomez-Herrero G, Atienza M, Egiazarian K, Cantero JL. Measuring directional coupling between EEG sources. Neuroimage. Nov.2008 vol. 43(no. 3):497-508. [PubMed: 18707006]

[23]. van Mierlo P, Carrette E, Hallez H, Vonck K, Roost DV, Boon P, Staelens S. Accurate epileptogenic focus localization through time-variant functional connectivity analysis of intracranial electroencephalographic signals. Neuroimage. 2011; vol. 56(no. 3):1122-1133. [PubMed: 21316472]

[24]. Cloostermans MC, de Vos CC, van Putten MJ. A novel approach for computer assisted eeg monitoring in the adult ICU. Clin. Neurophysiol. 2011; vol. 122:2100-2109. [PubMed: 21474372]

[25]. Nolte G, Ziehe A, Nikulin VV, Schlögl A, Krämer N, Brismar T, Müller K-R. Robustly estimating the flow direction of information in complex physical systems. Phys. Rev. Lett. Jun 14.2008 vol. 100(no. 23):234101. [PubMed: 18643502]

[26]. Granger CWJ. Investigating causal relations by econometric models and cross-spectral methods. Econometra. Aug.1969 vol. 37:424-438.

[27]. Implementation of PSI in MATLAB code. 2012. [Online]. Available: http://ml.cs.tu-berlin.de/causality/

[28]. Lee HC, van Drongelen W, McGee AB, Frim DM, Kohrman MH. Comparison of seizure detection algorithms in continuously monitored pediatric patients. J. Clin. Neurophysiol. 2007; vol. 24(no. 2):137-146. [PubMed: 17414969] 


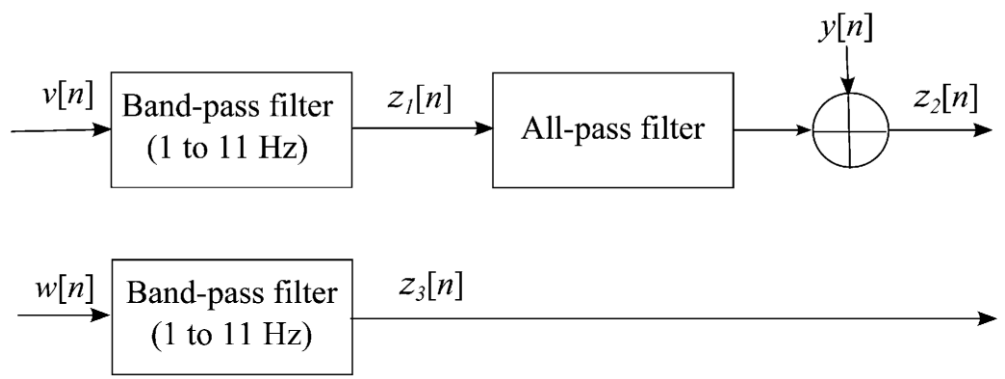

Fig. 1.

Simulation scenario for evaluating sensitivity of PSI to segment length. $v[n]$ and $w[n]$ are uniform $[-100,100]$ independent random variables, while $y[n]$ is a sequence of Gaussian distributed independent random variables with zero mean and variance 0.1 . The bandpass filter of $1-11 \mathrm{~Hz}$ passband is a 12th-order IIR filter and the allpass filter is a 3rd order IIR filter, both assuming a sampling frequency of $100 \mathrm{~Hz}$. The allpass filter introduces a nonlinear phase shift between $z_{1}[n]$ and $z_{2}[n]$. 


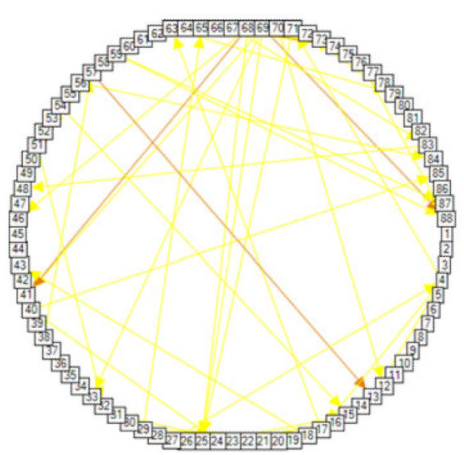

(a)

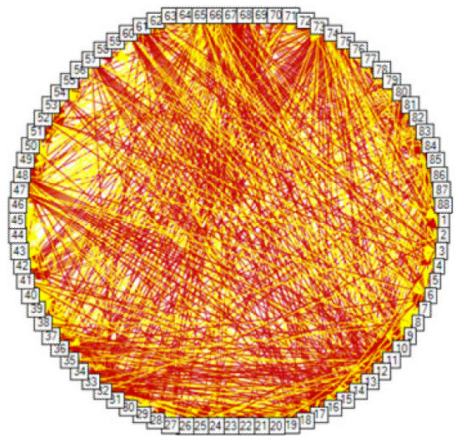

(b)

Fig. 2.

Graphical depiction of causal interaction between channels using the PSI metric $\Psi_{i j}$ with $i, j$ $=1,2, \ldots, 88$ during 60 -s segments for patient 2 . Only values with $\Psi_{i j}>2$ are shown. Yellow indicates smaller PSI values while red indicates larger PSI values. (a) Interictal segment. (b) Ictal segment. 


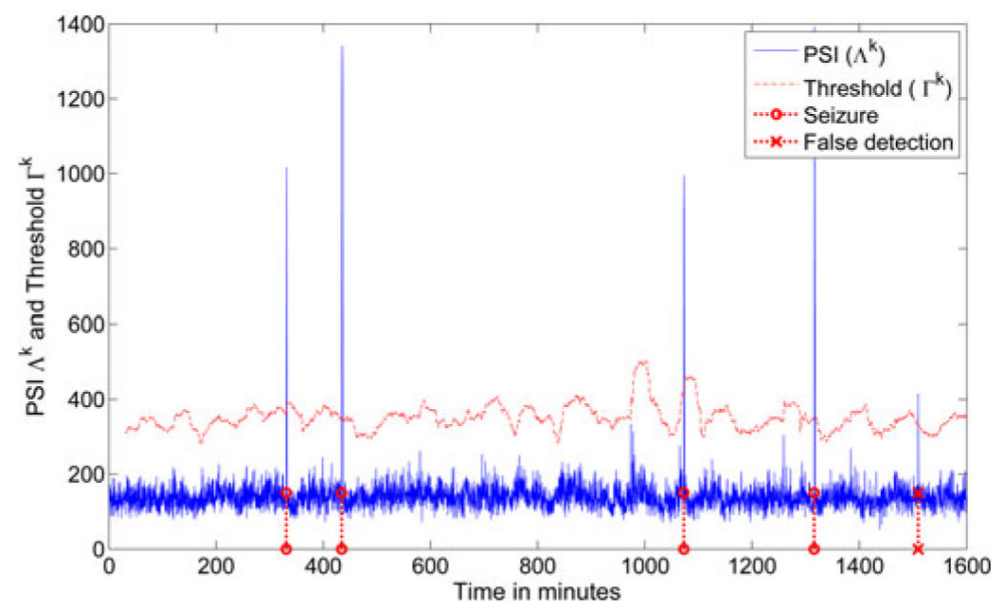

Fig. 3.

PSI metric $\Lambda^{k}[(7)$ computed with segment length $N=20 \mathrm{~s}]$ over time plotted with the threshold $\Gamma^{k}[(9)$ computed with $L=90$ and $c=8]$ for $1600 \mathrm{~min}$ of data for patient 1 . All four seizures were identified and one event is falsely declared as seizure. 

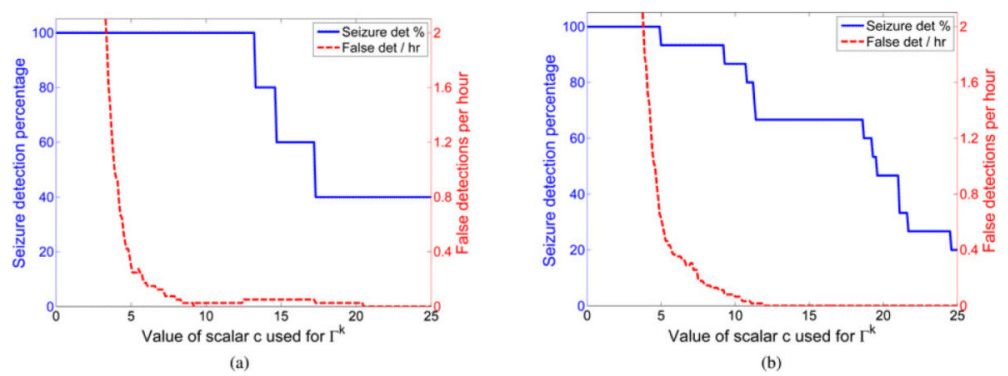

Fig. 4.

Seizure detection percentage (solid line) and false detections per hour (dashed line) for a range of values of $c$ in (9). Twenty-second segments were used in (4) and $L=90$ segments (30 min) was set in (9) to calculate the moving threshold $\Gamma^{k}$. (a) Patient 2. (b) Patient 5. 
(A)
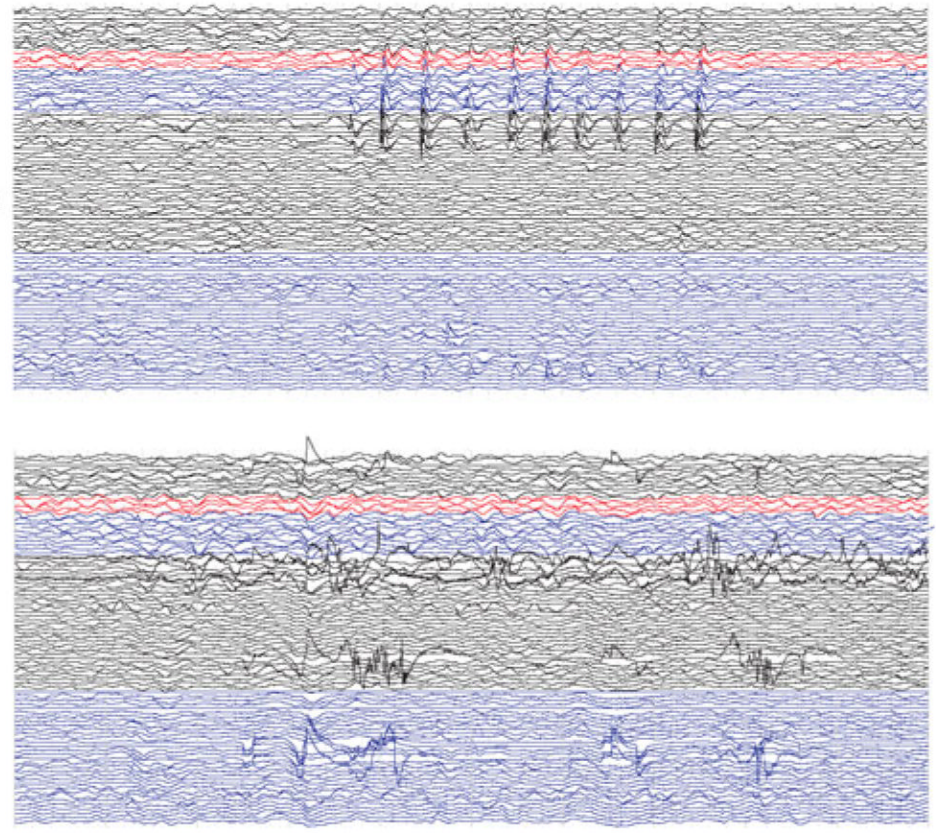

Fig. 5.

Shown are two $10 \mathrm{~s}$ segments of ECoG representative of PSI false detections, both taken from patient $3 \mathrm{~s}$ record. Panel A is an example of abnormal activity that occasionally organizes, but does not rise to the level of a clinical seizure and may therefore be considered a subclinical event. Panel B shows equipment artifact. 


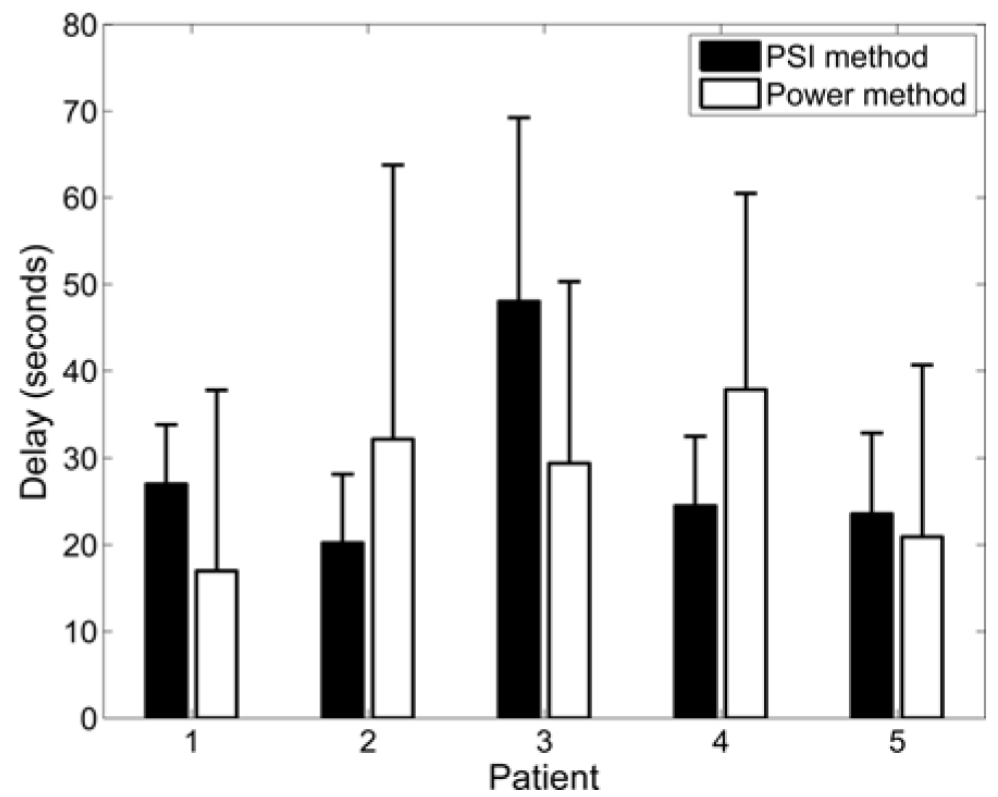

Fig. 6.

Average delay in detecting seizures using 20-s segments with a threshold $\Gamma^{k}$ that employs $L$ $=90$ (30 min) in (9). Error bars denote one standard deviation. The value of the constant $c$ in (9) was 4.8 for the PSI method and 2.1 for the power method. 

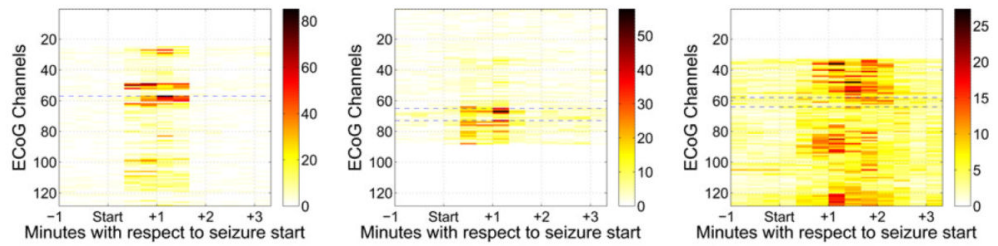

(a)
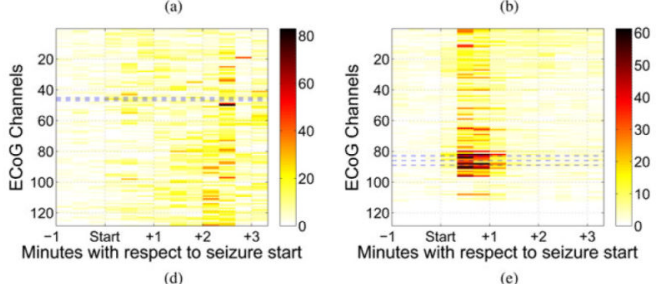

(c)

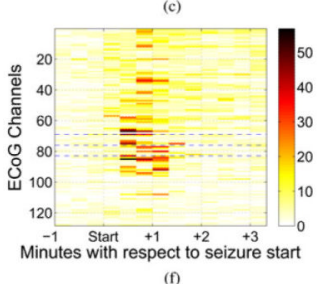

Fig. 7.

Plot of the average seizure evolution PSI $\tilde{\Lambda}_{i}^{l}$ defined in (13) using 20-s segment lengths for configurations with at least five seizures. (a) Patient 1. (b) Patient 2. (c) Patient 3. (d) Patient 4: second electrode configuration. (e) Patient 5: first electrode configuration. (f) Patient 5: second electrode configuration. The horizontal long dashed lines are the clinically identified leading channels. 


\begin{tabular}{|c|c|c|c|c|c|}
\hline 离 & 0 & in & 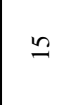 & 0 & 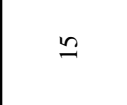 \\
\hline 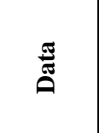 & 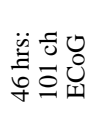 & 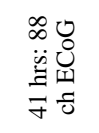 & 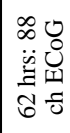 & 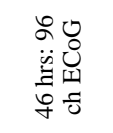 & 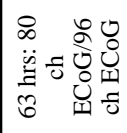 \\
\hline 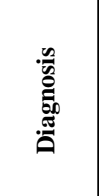 & 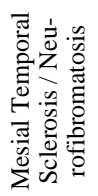 & 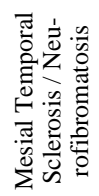 & 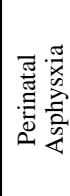 & 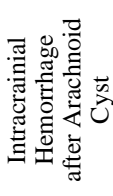 & 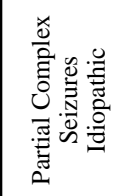 \\
\hline ڤે & 山 & 山 & $\Sigma$ & $\Sigma$ & $\Sigma$ \\
\hline 喿产产焉 & $\bar{N}$ & 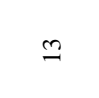 & $\simeq$ & $=$ & $\stackrel{m}{2}$ \\
\hline & - & $N$ & $m$ & 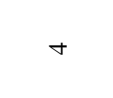 & in \\
\hline
\end{tabular}

IEEE Trans Biomed Eng. Author manuscript; available in PMC 2013 April 01. 


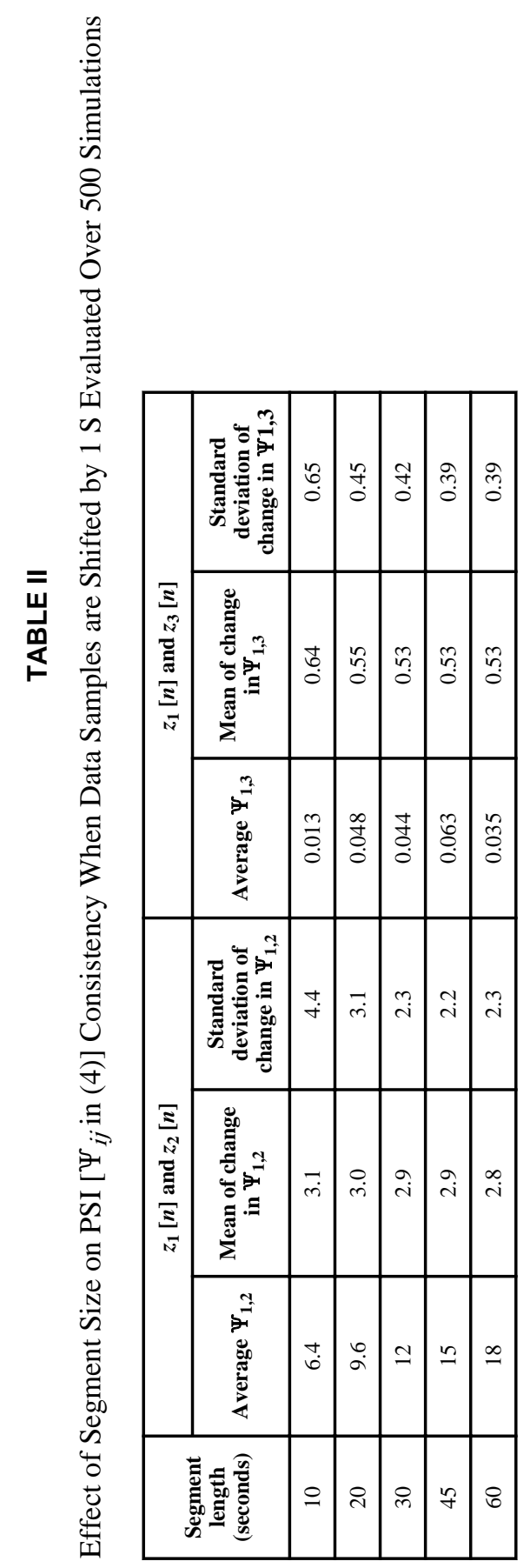

IEEE Trans Biomed Eng. Author manuscript; available in PMC 2013 April 01. 


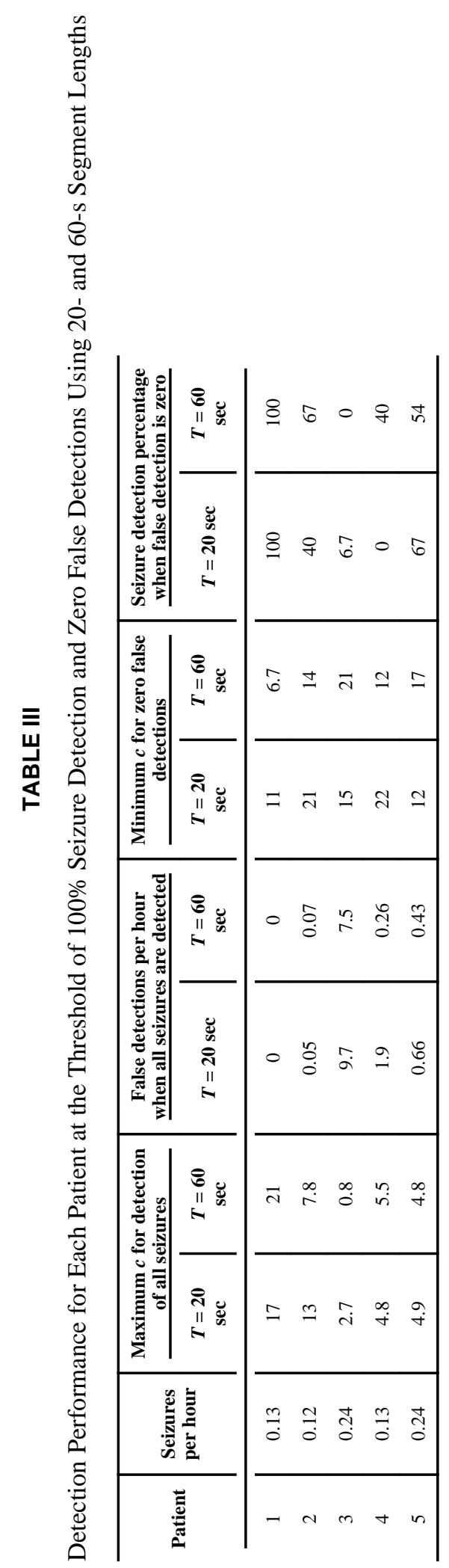

IEEE Trans Biomed Eng. Author manuscript; available in PMC 2013 April 01. 


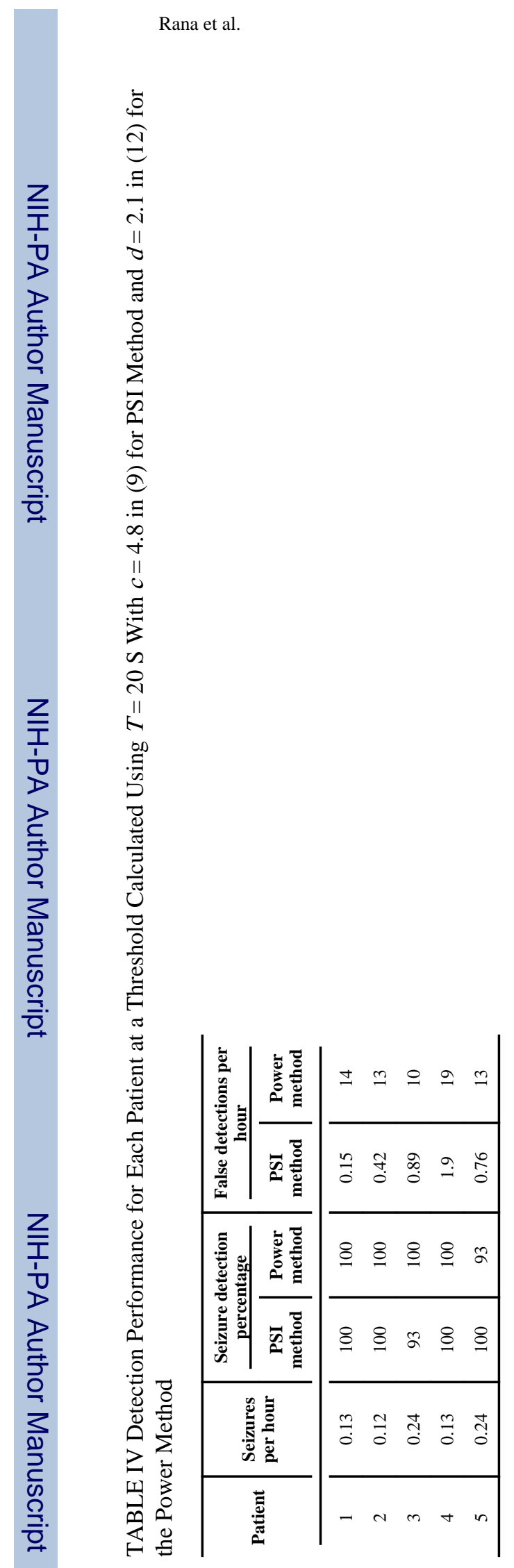

IEEE Trans Biomed Eng. Author manuscript; available in PMC 2013 April 01. 
TABLE V

Channels With the Largest Average PSI Outflow (13) Corresponding the Seizure Evolution Graphs in Fig. 7

\begin{tabular}{c|c|c}
\hline Patient & $\begin{array}{c}\text { Channels with five } \\
\sim \boldsymbol{l} \\
\text { largest } \begin{array}{c}\boldsymbol{\Lambda} \boldsymbol{i} \\
\text { (Equation (13)) }\end{array}\end{array}$ & $\begin{array}{c}\text { Clinically- } \\
\text { identified leading } \\
\text { channel numbers }\end{array}$ \\
1 & $49,50, \underline{57}, 60,52$ & 57 \\
2 & $67,76,74,68, \underline{65}$ & 65,73 \\
3 & $55,36,48,105,125$ & 58,64 \\
$42^{\text {nd }}$ electrode \\
configuration) \\
$\begin{array}{c}5 \text { (1 }{ }^{\text {st }} \text { electrode } \\
\text { configuration) }\end{array}$ & $50,87,124,80,19$ & $45,46,47$ \\
$\begin{array}{c}5 \text { (2 } \\
\text { configuration) }\end{array}$ & $85,88,91,90,80$ & $83,86,89$ \\
\hline
\end{tabular}

